\title{
PARSEVAL'S EQUALITY IN FUZZY NORMED LINEAR SPACES
}

\author{
BAYAZ DARABY, FATANEH DELZENDEH, and ASGHAR RAHIMI
}

\begin{abstract}
In this paper, we investigate Parseval's equality and define the fuzzy frame on Felbin fuzzy Hilbert spaces. For showing the importance of defining fuzzy frame, we know that, in the classical Hilbert space, $C(\Omega)$ is not normable, but, in this manuscript, we prove that $C(\Omega)$ (the vector space of all continuous functions on $\Omega$ ) is normable in a Felbin fuzzy Hilbert space and so the defining fuzzy frame on $C(\Omega)$ is possible. These consequences of the category of fuzzy frames in Felbin fuzzy Hilbert spaces are wider than the category of the frames in the classical Hilbert spaces.
\end{abstract}

MSC 2010. 54A40, 03E72

Key words. Fuzzy norm, fuzzy inner product space, fuzzy Hilbert space, fuzzy frame.

\section{REFERENCES}

[1] T. Bag and S.K. Samanta, Fuzzy bounded linear operators, Fuzzy Sets and Systems, 151 (2005), 513-547.

[2] T. Bag and S.K. Samanta, Finite dimensional fuzzy normed linear spaces, Journal of Fuzzy Mathematics, 11 (2003), 687-705.

[3] I. Beg and K.M. Aamir, Fuzzy Wavelets, Journal of Fuzzy Mathematics, 21 (2013), 623-638

[4] R. Biswas, Fuzzy inner product spaces and fuzzy norm functions, Inform. Sci., 53 (1991), $185-190$

[5] S.C. Cheng and J.N. Mordeson, Fuzzy linear operators and fuzzy normed linear spaces, Bull. Calcutta Math. Soc., 86 (1994), 429-436.

[6] O. Christensen, An introduction to frames and Riesz bases, Birkhäuser, 2003.

[7] B. Daraby, Z. Solimani and A. Rahimi, Some properties of fuzzy Hilbert spaces, Complex Anal. Oper. Theory, 11 (2017), 119-138.

[8] B. Daraby, Z. Solimani and A. Rahimi, A note on fuzzy Hilbert spaces, Journal of Intelligent Fuzzy Systems, 31 (2016), 313-319.

[9] B. Daraby, F. Delzendeh, A. Rostami and A. Rahimi, Fuzzy normed linear spaces and fuzzy frames, Azerb. J. Math., 9 (2019), 96-121.

[10] B. Daraby, A. Rostami and A. Rahimi, On the study of fuzzy Hilbert spaces by fuzzy isometric isomorphisms, TWMS Journal of Applied and Engineering Mathematics, to appear.

[11] I. Daubechies, Ten lectures on wavelets, SIAM, Philadelphia, 1992.

[12] I. Daubechies, A. Grossmann and Y. Meyer, Painless nonorthogonal expansions, J. Math. Phys, 27 (1986), 1271-1283.

The authors thank the referee for his helpful comments and suggestions.

DOI: 10.24193/mathcluj.2021.1.05 
[13] R.J. Duffin and A.C. Schaeffer, A class of nonharmonic Fourier series, Trans. Amer. Math. Soc., 72 (1952), 341-366.

[14] A.M. El-Abyad and H.M. El-Hamouly, Fuzzy inner product spaces, Fuzzy Sets and Systems, 44 (1991), 309-326.

[15] C. Felbin, Finite dimensional fuzzy normed linear spaces, Fuzzy Sets and Systems, 48 (1992), 239-248.

[16] A. Hasankhani, A. Nazari and M. Saheli, Some properties if fuzzy Hilbert spaces and norm of operators, Iran. J. Fuzzy Syst., 7 (2010), 129-157.

[17] A.K. Katsaras, Fuzzy topological vector spaces II, Fuzzy Sets and Systems, 12 (1984), $143-154$

[18] P. Majumdar and S.K. Samanta, On fuzzy inner product spaces, Fuzzy Mathematics, 16 (2008), 377-392.

[19] S. Mukherjee and T. Bag, Some properties of fuzzy Hilbert spaces, International Journal of Mathematics and Computing, 1 (2011), 50-56.

[20] M. Mursaleen, H.M. Srivastava and S.K. Sharma, Generalized statistically convergent sequences of fuzzy numbers, Journal of Intelligent \& Fuzzy Systems, 30 (2016), 15111518 .

[21] A. Popoola, S. Ahmad and K. Ahmad, A Fuzzy-Wavelet Method for Analysing NonStationary Time Series, in Proc. of the 5th International Conference on Recent Advances in Soft Computing, Nottingham, United Kingdom, 2004, 231-236.

Received May 22, 2019

Accepted November 3, 2019
University of Maragheh Department of Mathematics Maragheh, Iran

E-mail: bdaraby@maragheh.ac.ir E-mail: fatenehdelzende@yahoo.com

E-mail: rahimi@maragheh.ac.ir 